\title{
Fate of the native aorta after repair of acute type A dissection: a magnetic resonance imaging study
}

\author{
N R Moore, A J Parry, B Trottman-Dickenson, R Pillai, S Westaby
}

\begin{abstract}
Objective-To determine late patency of the aortic false lumen and propensity for aneurysm formation after repair of type $A$ dissection.

Design-Retrospective follow up study. Setting-Regional cardiac surgical unit. Patients -28 patients after repair of type A dissection.

Methods-Magnetic resonance imaging (MRI) was performed between 6 weeks and 12 months after operation.

Results-A patent distal false lumen with demonstrable blood flow was found in 22 patients $(78 \%)$. Only six patients had complete obliteration of the false lumen by thrombus. The conduct of operation did not influence this. Nine patients (32\%) showed aneurysmal dilatation of the false lumen and three had a repeat operation. Conclusions-So-called "successful repair" of aortic dissection does not obliterate the distal false lumen. MRI is a safe and effective radiological procedure for determining patency and dilatation in the false lumen. Patients with type $A$ dissection with blood flow in the false lumen should be studied every 6 months to look for aneurysmal dilatation.
\end{abstract}

(Heart 1996;75:62-66)

Keywords: magnetic resonance imaging; repair of type A dissection; patency of aortic false lumen; aneurysmal dilatation

Surgical treatment for acute type A dissection aims to prevent aortic rupture, restore competence of the aortic valve, and redirect blood into the true aortic lumen. In most patients this is achieved by replacement of the ascending thoracic aorta with resuspension of the commissures of the aortic valve. A few patients including those with Marfan's syndrome are treated with aortic root replacement. Nevertheless, cystic medial necrosis affects the whole of the aorta and its major branches and the aorta distal to the area of repair may contain further intimal tears. In most patients the false aortic lumen extends widely and the ultimate fate after repair is difficult to anticipate. Aneurysm formation occurs insidiously and may result in rupture and sudden death.

There is increasing evidence that in many patients the false lumen does not thrombose ${ }^{12}$ as is the principle behind medical treatment of acute type B dissection. Indeed, flow in the false lumen may be essential for perfusion of some abdominal viscera. ${ }^{2}$ Continued radiological assessment is important in asymptomatic patients to identify those at risk from aneurysms of the arch or thoracoabdominal aorta. The results of follow up by magnetic resonance imaging (MRI) in 28 patients following repair of acute type A dissection are described here. Our aim was to determine the incidence of patency of the false lumen and persistence of blood flow into the new channel. After late mortality (months) from aortic rupture in two patients who had type A dissection repair, we elected to follow up others by MRI in an effort to determine the propensity for this lethal event.

\section{Patients and methods}

Between October 1987 and October 1991, 55 patients underwent repair of acute type A dissection, five of whom had Marfan's syndrome. There were 38 men and 17 women, aged 25-81 (mean 59.5) years. The clinical diagnosis of acute aortic dissection was confirmed before operation by computed tomography (CT), aortography, or latterly, transoesophageal echocardiography. The dissection was acute in all patients and none had existing aneurysms of the aortic arch or descending thoracic aorta. In addition, the false lumen extended through the descending aorta to the diaphragmatic hiatus or beyond in all patients.

Two different surgical methods were used. Between 1987 and 1989, the perfusion temperature was reduced to $28^{\circ} \mathrm{C}$ and the aorta cross clamped just proximal to the innominate artery. The ascending aorta was opened, the dissection identified and excised, and the aorta replaced with a Hemashield (Meadox (UK), Caddington, UK) graft. If the intimal tear was not found within the ascending aorta, then the systemic core temperature was reduced to $18^{\circ} \mathrm{C}$ for a period of circulatory arrest. The aortic arch tear was excised and the arch replaced. During the second period between 1989-1991, the open ended repair technique described by Cooley and Livesay $^{3}$ was adopted. After repair of the aortic root and valve all distal graft anastomoses were undertaken with hypothermic circulatory arrest for direct vision of the aortic arch. This allowed excision of the clamp site (applied during repair of the aortic root) and prevented the risk of cross clamp damage to the aorta beyond the distal graft anastomosis which 
Table 1 Patients who did not undergo magnetic resonance imaging $(M R I)$

\begin{tabular}{ll}
\hline & $n$ \\
\hline $\begin{array}{l}\text { Early mortality } \\
\quad(30 \text { days) }\end{array}$ & 9 \\
Late mortality & 6 \\
$\begin{array}{l}\text { Ferromagnetic valve } \\
\text { prosthesis }\end{array}$ & 5 \\
Pacemaker & 2 \\
Too obese & \\
$\quad$ for scanner & 1 \\
Declined MRI & 4 \\
\hline
\end{tabular}

^One was old, two were claustrophobic, and one refused to travel long distance.

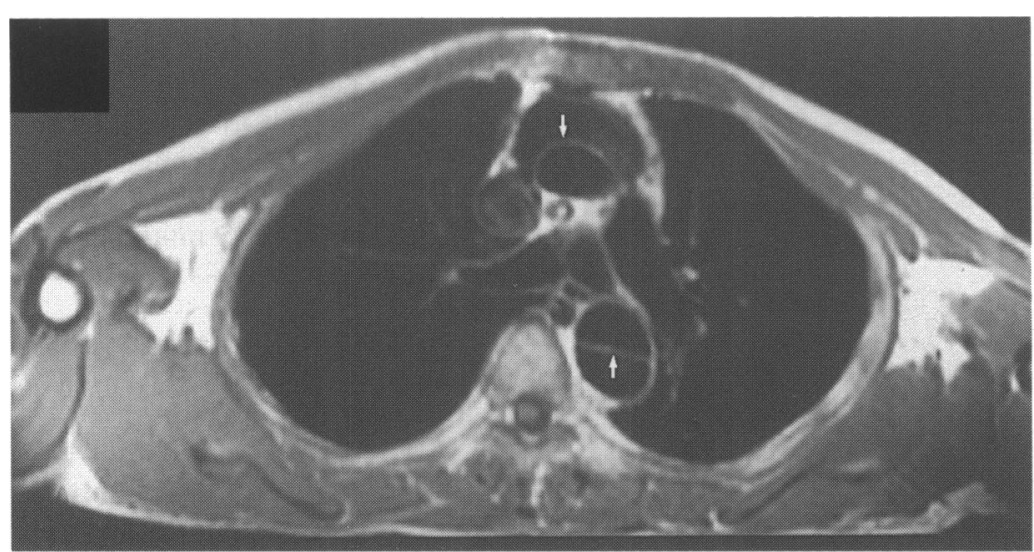

Figure 1 Transverse $T 1$ weighted image through the ascending aorta cranial to the graft, showing a residual type $A$ dissection (arrows) with patent false lumen.

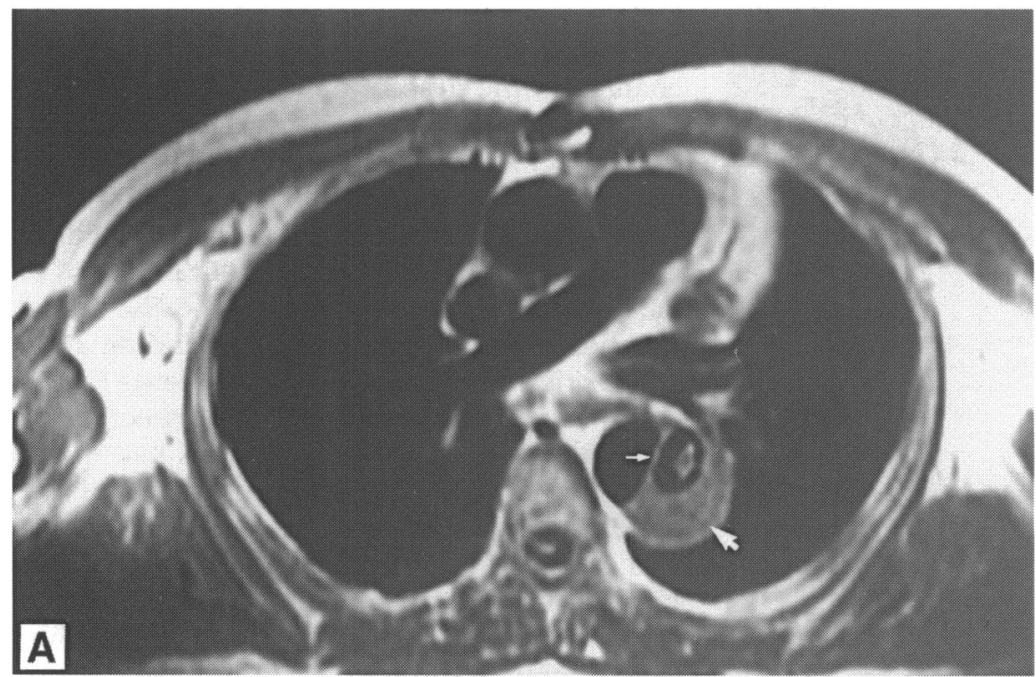

Figure 2 (A) Transverse and $(B)$ coronal $T 1$ weighted images. The graft seen in $(A)$ is satisfactory. There is a residual dissection of the arch and descending aorta (arrows) with intermediate signal thrombus partially occluding the false lumen. existed with our earlier method. In this second period gelatine resorcinol formol glue ${ }^{4}$ was used to eliminate the false lumen at the anastomotic sites and increase the suture holding capacity of the damaged tissue. For patients with Marfan's syndrome and others who required aortic root replacement with either method of distal repair, the aortic valve was excised and the coronary ostia mobilised then reimplanted into a valved conduit.

\section{CLINICAL OUTCOME}

Of the 55 patients, 13 (24\%) underwent aortic root replacement with a valved conduit, whereas 42 had ascending aortic replacement with a Hemashield graft and repair of the aortic valve. Patients who had aortic root or ascending aortic replacement were evenly distributed between the two surgical methods described. Four patients required aortic arch replacement for an arch tear; this comprised resection and replacement of the underside of the aortic arch in three patients, while one 84 year old woman had complete aortic arch replacement with reimplantation of the head and neck vessels.

There were no operative deaths, but nine patients died within 30 days of surgery (early mortality $16 \%$ ). Five of these patients presented late (more than $60 \mathrm{~h}$ after acute dissection) with established multisystem failure from which they did not recover postoperatively. Two others died of mensenteric vascular occlusion and bowel infarction. Two patients died out of hospital from free rupture of the patent false lumen.

Clinical follow up in the 46 surviving patients varied from 6 months to 5 years. MRI was performed in 28. Table 1 gives the reasons for exclusion for MRI in the 18 remaining patients. Six patients died suddenly of suspected cardiovascular events during the follow up period (late mortality 13\%). One patient who had aortic root replacement with a mechanical valved conduit was anticoagulated and suffered a fatal stroke. The remaining five patients had uneventful ascending aortic replacement with valve repair. Postmortem examination in one showed a ruptured descending thoracic aorta but necropsy was not performed on the remaining five.

CONDUCT OF MAGNETIC RESONANCE IMAGING MRI scans were performed between 6 weeks and 12 months after surgery and repeated every 6 months in patients with signs of aneurysm formation. Imaging was performed using a MRI system (Signa Advantage IGE Medical Systems, Slough, UK) operating at $1.5 \mathrm{~T}$. Cardiac and respiratory motion induced artefacts were reduced by the use of acquisitions gated to the $R$ wave of the electrocardiogram and respiratory compensation respectively. T1 weighted spin echo images were obtained in the coronal, transverse, and where indicated sagittal or oblique planes from neck to mid-pelvis and these were supplemented by further $\mathrm{T} 2$ weighted spin echo images where appropriate. Section thickness was either $8 \mathrm{~mm}$ with a $2 \mathrm{~mm}$ intersection gap or $10 \mathrm{~mm}$ with a $5 \mathrm{~mm}$ intersection gap for spin echo acquisitions. The acquisition matrix

Table 2 Results of the magnetic resonance imaging (MRI) studies $(n=28)$

\begin{tabular}{lr}
\hline & $n$ \\
\hline Complete obliteration of the false lumen & $6(21)$ \\
Patency in the aortic arch & $22(79)$ \\
Patency in the brachiocephalic vessels & $16(57)$ \\
Patency in the descending thoracic aorta & $17(61)$ \\
Patency in the thoracoabdominal aorta & $15(54)$ \\
\hline
\end{tabular}

Values in parentheses are percentages. 
Figure 3 Sagittal T1 image through the graft in is a dissection flap extending into the innominate artery (arrow). the ascending aorta. There
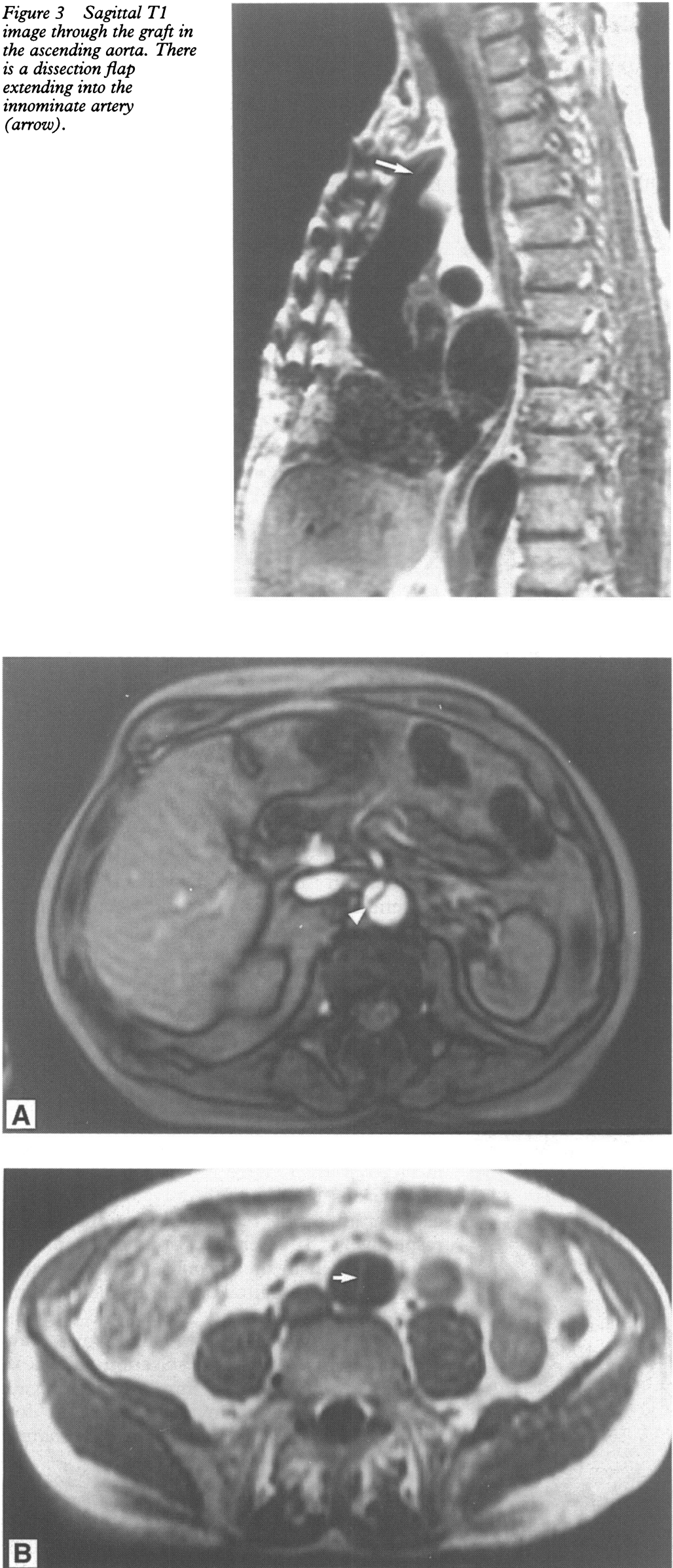

Figure 4 (A) Transverse gradient recalled acquisition in the steady state (GRASS) image at the origin of the superior mesenteric artery showing high intensity within flowing blood. The dissection flap is seen as a low signal curved stripe (arrowhead). (B)

Transverse T1 image in the same patient just above the bifurcation of the aorta showing the residual dissection (arrow). was $256 \times 128$ for the coronal images, and $256 \times 192$ for the transverse, sagittal, and oblique images. The field of view varied according to patient size. In addition, a gradient recalled acquisition in the steady state (GRASS) image was performed in the transverse plane, as either a single slice technique or a four section, 16 partition, cine acquisition. The flip angle for the GRASS sequences was $30^{\circ}$ and the acquisition matrix was $256 \times$ 128. The GRASS or cine GRASS studies were performed to confirm the spin echo assessment of luminal patency or thrombosis.

A patent false lumen was typified by signal void on spin echo images and variations of high signal intensity on the GRASS images. Material of constant signal intensity on both pulse sequences was diagnosed as thrombus. Slowly flowing blood showed intermediate signal intensity on $\mathrm{T} 1$ but variations in high signal intensity on the cine GRASS images.

\section{Results}

Of the 28 patients who underwent MRI, 22 $(78 \%)$ had some demonstrable blood flow within the false lumen (figs 1 and 2). Only six patients had complete obliteration of the false lumen by thrombus (table 2). Neither the overall time from the initial operation nor the type of surgical repair influenced persistence of blood flow within the false lumen. Extension of the surgical procedure into the aortic arch did not prevent patency of the false channel in the descending thoracic aorta. The false lumen was present in the arch in 22 patients $(79 \%)$ and extended widely into the brachiocephalic vessels, and descending and thoracoabdominal aorta. One patient developed a dissection of the aortic root proximal to the ascending aortic graft despite the initial tear being located high in the ascending aorta at the original operation. In 16 patients $(57 \%)$ the brachiocephalic arteries were dissected (fig 3), although none of these patients had manifest neurological signs or symptoms. Similarly, none of the 17 patients with persistent dissection of the descending thoracic aorta $(61 \%)$ or 15 with thoracoabdominal involvement $(54 \%)$ (fig 4) was symptomatic. One patient with thoracoabdominal dissection had a normal aortic arch on imaging but an apparently new dissection (type B) in the descending aorta.

As well as persistent blood flow in the false lumen, nine patients (32\%) including all those with Marfan's syndrome showed aneurysmal dilatation (fig 5). The size of aneurysm ranged from 5.0 to $9.3 \mathrm{~cm}$, and elective reoperation was undertaken in three patients because of aneurysm size greater than $6.0 \mathrm{~cm}$. The first patient had aortic root replacement for aneurysmal sinuses of Valsalva. The second had complete replacement of the aortic arch for an $8.7 \mathrm{~cm}$ aneurysm (fig 6 ) with tracheal compression. The last patient who had Marfan's syndrome underwent repair of a massive descending thoracic aneurysm and suffered paraplegia followed by fatal multisystem organ failure. 


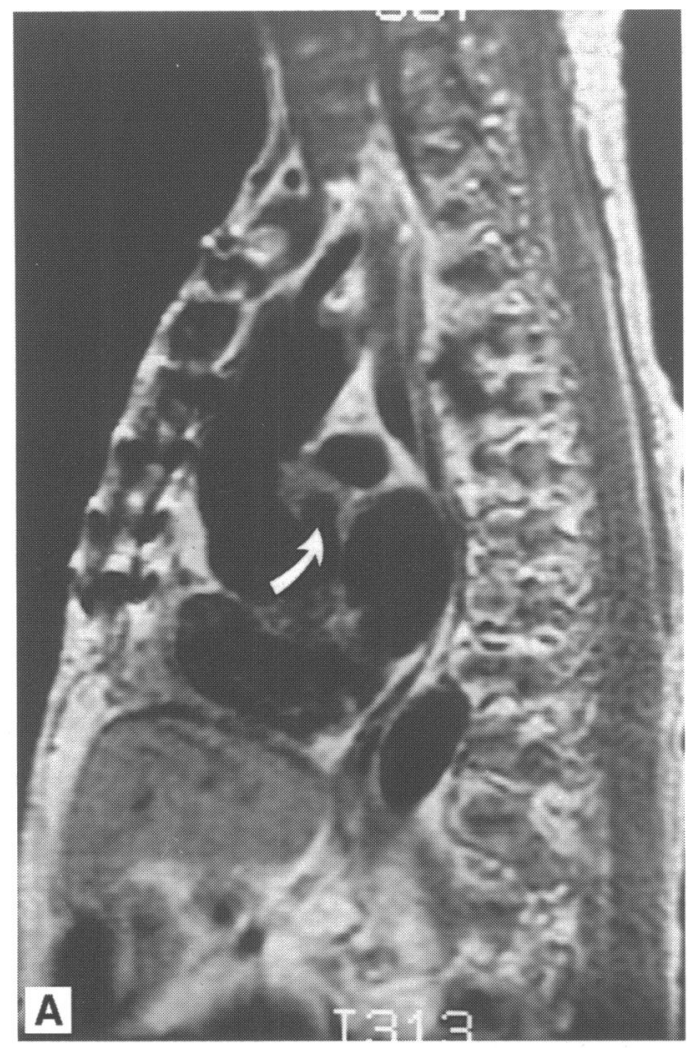

\section{Discussion}

Surgery for acute type A aortic dissection aims to redirect blood flow into the true aortic lumen, which theoretically should allow the false channel to thrombose and obliterate. In practice, patients who survive primary operation remain at significant risk of death from rupture of other areas particularly the descending aorta. Rupture of the distal aorta accounts for about $30 \%$ of deaths after repair of type A dissection and up to $30 \%$ of patients develop aneurysms distal to the ascending aortic graft (16\% in our series). ${ }^{5}$ In the acute phase transoesophageal echocardiography is the preferential method to diagnose type A dissection in a haemodynamically unstable patient and is used to demonstrate the site of tear. ${ }^{6}$ Although MRI has been shown to be highly sensitive and selective in the diagnosis of acute aortic dissection, ${ }^{6-8}$ we consider this procedure to be impractical in this situation. ${ }^{1}$ The time required in the magnet is too long for an unstable patient with acute aortic regurgitation or cardiac tamponade. At follow up, however, MRI is superior to other techniques including contrast enhanced CT, transthoracic or transoesophageal echocardiography or aortography (sensitivity $96-100 \%$, and specificity of $98-100 \%$ MRI $v 83-94 \%$ for CT). ${ }^{6} 910$ Furthermore, CT requires the use of large

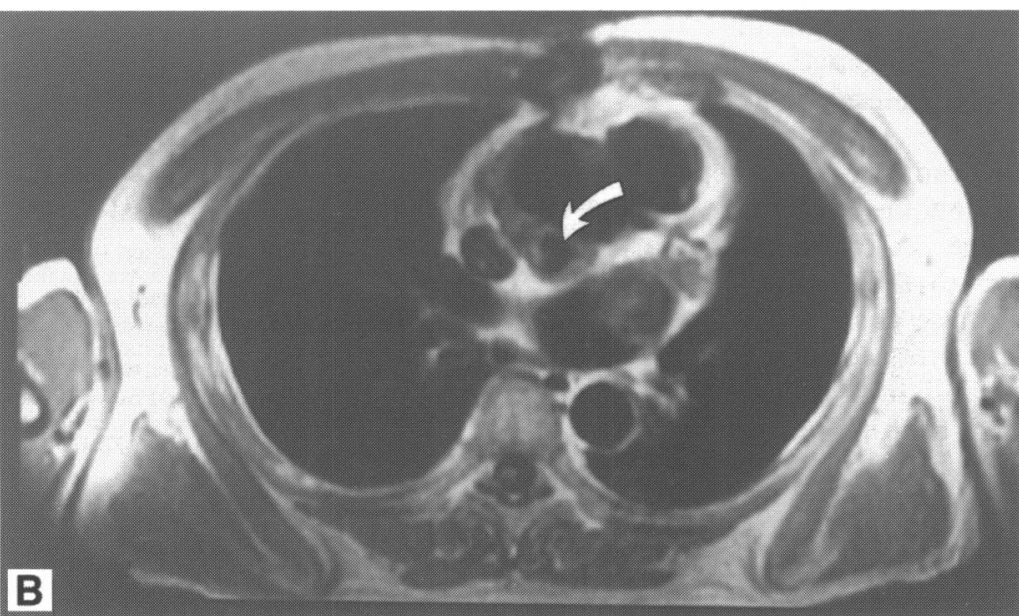
doses of iodinated contrast medium and incurs a substantial radiation dose to the patient. MRI is less invasive than transoesophageal echocardiography on an outpatient basis, and provides important information relating to flow patterns within the true and false aortic lumen ${ }^{11}$ together with a realistic assessment of the development of thrombus. ${ }^{12}$ It provides accurate information about the degree of dilatation in the false lumen, ${ }^{13}$ which when associated with blood flow seems to be a bad prognostic sign. ${ }^{14}$ However, the proportion of patients excluded from MRI is significant. The problem of ferromagnetic substances in valve prostheses is now less with the pyrolitic carbon bileaflet valves. The issue of long distance

Figure 5 (A) Sagittal and (B) transverse T1 weighted images in a patient with a partially thrombosed aneurysm of the non-coronary sinus (curved arrow) (same patient as in fig 1). travel will disappear with increasing availabil-

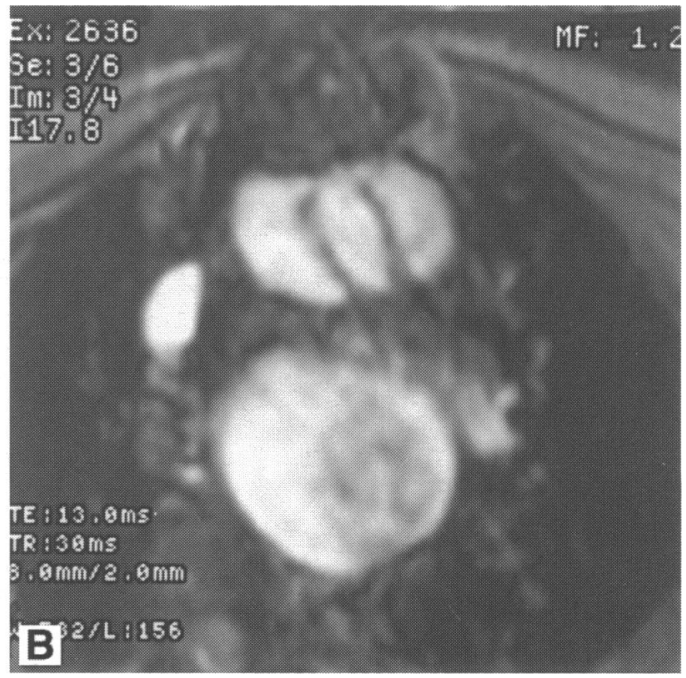

Figure 6 (A) Transverse $T 1$ weighted and (B) cine gradient recalled acquisition in the steady state (GRASS) images showing a residual ascending aortic dissection and an $8.7 \mathrm{~cm}$ aneurysm of the proximal descending aorta. The intermediate signal on the T1 weighted image (arrow) ( $A$ ) is of high signal on the GRASS image (B) indicating turbulent, slowly flowing blood. 
ity of MRI scanners but the problem of claustrophobia remains.

In our patients there was a high incidence of patency and blood flow within the false lumen irrespective of whether or not the open ended technique was used for repair. None of our three patients who had a repeat operation had symptoms sufficient to warrant surgery but MRI showed large thin walled aneurysms. None of these aneurysms was present before operation as imaged by CT, aortography or transoesophageal echo. Evidence from the natural history of atherosclerotic abdominal aortic aneurysms shows that the incidence of rupture is $80 \%$ when the aneurysm is $>8 \mathrm{~cm}$ in diameter, $43 \%$ when $>6 \mathrm{~cm}$ and $4 \%$ when $<5 \mathrm{~cm} .{ }^{15}$ No report has yet addressed the relative risk of rupture in aneurysms of the false lumen after aortic dissection but it is unlikely that extrapolation from atherosclerotic aneurysms is useful.

Patients with Marfan's syndrome are particularly at risk from late aortic aneurysm after aortic dissection and every effort should be made to replace the Marfan aortic root before dissection occurs. ${ }^{16}$ Surgery is therefore recommended for aortic root dilatation of $6.0 \mathrm{~cm}$ irrespective of the degree of aortic regurgitation. ${ }^{17}$ This dimension should be reduced to $5.0 \mathrm{~cm}$ for those with a family history of dissection as the mortality for elective root replacement is less than $5 \%$ in experienced hands. ${ }^{16}$

We conclude that MRI scanning is a safe and effective method for follow up of patients after repair of acute type A dissection. Attention should be drawn to the extremely high rate of patency of the false channel even after successful ascending aortic replacement with uneventful recovery. This may progress to aneurysm formation and rupture particularly in patients with Marfan's syndrome. Because of this we recommend follow up every 6 months by MRI for patients who survive surgery for acute type $A$ dissection and elective surgery for those with aneurysms $(>6.0 \mathrm{~cm})$.

1 Erbel R, Odat H, Meyer J, et al. Effect of medical and surgical therapy on aortic dissection evaluated by transoesophageal echocardiography-implication for prognosis and therapy. Circulation 1993;87:1604-15.

2 Ayala K, Chandrasekeran K, Otto T, et al. Are the Doppler characteristics of the entry site of an intimal flap useful in the evaluation and follow up of aortic dissection? [abstract] $\mathcal{F}$ Am Coll Cardiol 1991;19:279.

3 Cooley DA, Livesay JS. Technique of "open" distal anastomosis for ascending and transverse arch resection. Cardiovasc Dis 1981;8:421-6.

4 Guilmet D, Bachet J, Goudot B, et al. Use of biological glue in acute aortic dissection. $\mathcal{F}$ Thorac Cardiovasc Surg glue in acute aor

5 DeBakey ME, McCollum CH, Crawford ES. Dissection and dissecting aneurysms of the aorta: twenty-year follow up of five hundred and twenty-seven patients treated surup of five hundred and twenty-seven

6 Nienaber CA, von Kidolitsch Y, Nicolas V, et al. The diagnosis of thoracic aortic dissection by non-invasive imaging procedures. $N \mathrm{Engl} \mathcal{F} \mathrm{Med} 1$ 1993;328:1-9.

7 Nienaber CA, Spielmann RP, von Kodolitsch Y, et al. Diagnosis of thoracic aortic dissection: magnetic resonance imaging versus transoesopheal echocardiography. Circulation 1992;85:434-47.

8 Kersting-Sommerhoff BA, Higgings CB, White RD, Sommerhoff CP, Lipton MJ. Aortic dissection: sensitivity and specificity of MR imaging. Radiology 1988;166: 651-5.

9 Erbel R, Engberding R, Danial W, Roelandt J, Visser C, Rennollet $\mathrm{H}$. Echocardiography in diagnosis of aortic dissection. Lancet 1989;1:457-61.

10 Vasile N, Matthiew D, Keita K, Lellouche D, Bloch G, Cachera JP. Computed tomography of thoracic aortic dissection: accuracy and pitfalls. $\mathcal{f}$ Comput Assist Tomogr dissection: accuracy

11 Petasnick JP. Radiologic evaluation of aortic dissection. Radiology 1991;180:297-305.

12 White RD, Ullyot DJ, Higgings CB. MR imaging of the aorta after surgery for aortic dissection. Am $\mathcal{F}$ Radiol 1988;150:87-92.

13 Galloway AC, Colvin SB, Grossi EA, et al. Surgical repair of type A aortic dissection by the circulatory arrest-graf inclusion technique in sixty-six patients. $\mathcal{f}$ Thora Cardiovasc Surg 1993;105:781-8.

14 Roberts CS, Roberts WC. Aortic dissection with the entrance tear in the descending thoracic aorta. Analysis of 40 necropsy patients. Ann Surg 1991;213:356-68.

15 Cronenwelt JL, Murphy TF, Zelerock GB, et al. Actuarial analysis of variables associated with rupture of smal abdominal aortic aneursysms. Surgery 1985;98:477-83.

16 Gott VL, Pyeritz RE, Cameron DE, Greene PS, McKusick VA. Composite graft repair of Marfan aneurysms of the VA. Composite graft repair of Marfan aneurysms of the 1991;52:38-45.

17 Pyeritz RE. Predictors of dissection of the ascending aorta in Marfan's syndrome. Circulation 1991;84(suppl II): 11-351. 